Mediterránea Ser. Biol. (1992/93), n. 14. Pág. 57-78

\title{
LOS BOSQUES PROTECTORES DE PINUS HALEPENSIS EN LA PROVINCIA DE ALICANTE. CARACTERÍSTICAS DE UN PROCESO DE REVEGETACIÓN.
}

\author{
por \\ ANTONIO PASTOR-LÓPEZ* Y JOAQUÍN MARTÍN-MARTÍN*
}

\section{RESUMEN}

La repoblación forestal con Pinus halepensis en la provincia de Alicante se analiza como método casi exclusivo de regeneración de la cubierta vegetal durante el período 1945-1985, sobre la base de un estudio de 120 estaciones de muestreo y de la documentación disponible en la Unidad Forestal Provincial.

Se describe el proceso en base a sus objetivos y metodología para conseguirlos. Se abordan los aspectos que definen la realidad de la repoblación, respondiendo a las preguntas ¿Cómo? ¿Cuándo? y ¿Dónde? se repuebla. Se describe la información disponible así como la necesaria a fin de facilitar su investigación y gestión.

Destaca la naturaleza protectora de estas masas y como consecuencia la escasez de información sobre las mismas. Aspectos relativos a la evolución temporal del proceso, la naturaleza de la roca madre, métodos de preparación del terreno y densidad de plantación son detallados, evidenciándose la necesidad de un sistema de información geográfica como herramienta básica para sistematizar y analizar la información de lo que debe considerarse como el mayor experimento ecológico en extensión a que se han sometido los ecosistemas afectados. Se discuten las necesidades y limitaciones a la hora de gestionar esta realidad, a fin de obtener el máximo beneficio de este proceso de repoblación.

PALABRAS CLAVE: Pinus halepensis, Repoblación forestal, Revegetación, Alicante, Cuenca mediterránea.

\section{SUMMARY}

Afforestation with Pinus halepensis in Alicante province was analyzed, as the only method for vegetation cover restoration, during the period 1945-1985, on the basis of 120 stands studied and the files available in the Unidad Forestal of the province.

\footnotetext{
* Dep.Ecología. Facultad de Ciencias.Universidad de Alicante.
} 
The process is described in relation to its objectives and their implementation. The answers to the questions How, Where and When is the afforestation done? define the process. The information available and that needed to allow research as well as management of these ecosystems is defined.

There was a clear lack of information due to the protective nature of most of these stands. Detailed descriptions on evolution in time of the process, bedrock of the areas, site preparation and density of plantation are given. The need for applying GIS techniques is emphasized to analyze and structure the information of what can be considered the largest ecological experiment -in area- that these ecosystems have suffered. The needs and limitations for managing this reality is discusssed in order to obtain the maximum benefit of this afforestation process.

KEYWORDS: Pinus halepensis, Afforestation, Restoration, Alicante, Mediterranean Basin.

\section{INTRODUCCIÓN}

Los procesos de repoblación forestal han sido una de las actividades centrales que los servicios forestales han desarrollado en la provincia de Alicante y en buena parte del Levante español durante los últimos cincuenta años. El término repoblación forestal, usado coloquialmente, representa para la mayoría de la población una actividad de plantación, generalmente de especies arbóreas, con la finalidad de mejorar la calidad estética de un determinado paisaje así como de proteger el suelo contra la erosión a medio y largo plazo. Este proceso es percibido ampliamente como una acción que mejora la realidad vegetal de una determinada zona y por extensión la de los organismos dependientes de ella, aunque esto no resulta siempre evidente cuando se analizan dichas actuaciones de una forma más detallada y sistémica.

El objetivo de este articulo es analizar los procesos de repoblación forestal en la provincia de Alicante, definiendo las razones que los motivan, los mecanismos de su puesta en práctica y los resultados preliminares obtenidos.

La razón que justifica este análisis ha sido la modificación antrópica que para multitud de ecosistemas representa este proceso. Claramente, es la única vía de revegetación artificial utilizada de forma extensiva por lo que afecta a importantes superficies.

Para definirlo es necesario conocer por qué, cómo, dónde y cuándo se ha realizado el proceso objeto de análisis, la creación de bosques protectores.

Entendiendo por ello, un tipo de revegetación que emplea especies arbóreas introducidas como plantones a densidades definidas y con métodos de preparación del terreno que se modifican en función de las características topográficas del substrato y de la infraestructura disponible con que se realizan.

Entendemos "bosque" en el sentido indicado por SPURR \& BARNES (1980) como "una de las formas de vida básicas a nivel fisionómico en que las comunida- 
des biológicas pueden clasificarse, caracterizada por el predominio de vegetación leñosa sustancialmente más alta que el hombre". De igual modo entendemos protectores desde el punto de vista selvícola (GARCÍA SALMERÓN, 1991), como aquellas formaciones que no tienen como objeto primordial la producción de madera o de materias para su explotación posterior en un ciclo temporal definido. Por el contrario, su función primordial suele ser la de proteger el suelo frente a procesos erosivos, la constitución de zonas de uso recreacional o la generación de ambientes que puedan ser colonizados por comunidades animales como vías de incrementar la superficie disponible para sus poblaciones y con una perspectiva conservacionista. Completamente fuera de sus objetivos pero entre sus efectos sociales está la creación de puestos de trabajo en el sector primario.

Estos tres objetivos reflejan en orden de importancia las causas que han motivado la repoblación en la provincia de Alicante. Sin lugar a dudas, la primera de ellas ha centrado la atención y ha justificado el diseño y realización de toda la actividad repobladora en la provincia (Muñoz J., 1986).

Desde una perspectiva administrativa, y habiendo definido el problema de proteger el suelo frente a la erosión como primordial, la solución dada debía de probar ser la más eficiente. La necesidad de cumplir esta condición se acrecienta al considerar que el producto interior bruto del sector forestal en la Comunidad Valenciana no alcanza más que el $0.4 \%$ de la actividad agrícola y ocupa el $40 \%$ del territorio.

El empleo de pino carrasco (Pinus halepensis) está justificado por su gran capacidad de desarrollo en condiciones de suelos esqueléticos y condiciones climáticas mediterráneas de elevado stress hídrico. No obstante, la variedad de ambientes y comunidades vegetales sobre las que se ha actuado genera cuestiones en lo referente a su idoneidad en todas ellas.

Es evidente que su empleo se apoya en características tales como la facilidad de su reproducción, su presencia como especie pionera en zonas próximas a las áreas de introducción, su resistencia en lo que a manejo de preplantación se refiere, y su considerable incremento en cobertura en las fases iniciales de su desarrollo (BAEZA M.J. et all. 1991). Todos estas características que justifican su empleo para cualquier técnico forestal no se han comprobado experimentalmente.

De igual modo tampoco se han realizado estas comprobaciones para otras especies alternativas. Esta tarea no es sencilla ni carente de costo y es normalmente la razón que se alude para explicar por qué se carece de dicha información. Sin embargo, es sorprendente que simultáneamente a los procesos de repoblación no se hayan establecido parcelas de control de la vegetación existente inicialmente o de otras especies dominantes en la flora local, a fin de disponer de evidencias "in situ" y sin dedicación de recursos. 
Desde un punto de vista estrictamente ecológico, estas actuaciones pueden tener distintas consecuencias según las características bióticas y abióticas de las zonas a restaurar. Las limitaciones económicas condicionan frecuentemente la posibilidad de analizar los sistemas preexistentes, pero es necesaria la definición de la estructura, de los regímenes de perturbación y resilencia de los sistemas sobre los que se interviene, para una gestión fundamentada en el conocimiento de los procesos que tienen lugar. Como señalan SPURR \& BARNES (1980) es necesario tener más información del sistema cuanto mayor es la intervención que se quiere hacer sobre él.

El establecimiento de parcelas control adecuadamente caracterizadas y con un sistema estandarizado de recopilación de la información en bases de datos incorporables a un sistema de información geográfica es imprescindible.

La definición de posibles estrategias de revegetación que cumpliendo los requerimientos de protección del suelo permitan el mantenimiento de los ecosistemas existentes así como de su reconstitución es tarea del ecólogo. No obstante, la definición de las limitaciones impuestas por los recursos a emplear para que la acción revegetadora sea factible es una labor a definir por el forestal.

El interés de mantener los procesos de revegetación en su línea actual o su modificación debe ser el resultado de una estrecha colaboración que examine las diferentes alternativas y defina procedimientos tanto de realización como de evaluación de futuras actuaciones.

Este trabajo aporta información sobre la realidad del proceso de revegetación realizado hasta mediados de la década de los 80 . Aunque sería nuestro deseo que dicha descripción resaltara todos los mecanismos que diferencian esta actividad de la acción estrictamente natural, la limitación de los datos disponibles en los servicios forestales condiciona nuestros resultados. Por otra parte, la dificultad de disponer de una cartografía detallada de la localización y evolución del proceso impide en este momento que muchas de las cuantificaciones se puedan realizar sobre el conocimiento areal. La aplicación de nuevas tecnologías como los sistemas de información geográfica, con gran plasticidad y potencialidad en el análisis espacial, representan una herramienta que no debe dejarse de incorporar en este proceso. Si las limitaciones provocadas por la carencia de bases cartográficas adecuadas han favorecido la no recolección de gran cantidad de información sobre la historia de este proceso hasta el momento, esto no debe seguir ocurriendo. Sin duda alguna, el proceso de repoblación forestal puede convertirse en el mayor experimento ecológico que se ha llevado a cabo en la historia reciente de los ecosistemas de nuestra zona.

Su potencialidad está por explotar pero indudablemente no se debe de perder esa oportunidad. 


\section{ZONA DE ESTUDIO}

El área considerada está comprendida entre las coordenadas 38-39² latitud norte y $0-1^{\varrho}$ longitud oeste, dentro de la provincia de Alicante, más concretamente circunscrita a los montes de propiedad estatal, de utilidad pública y consorciados. Estas zonas representan menos del $50 \%$ de las zonas con vegetación natural en el Mapa de Cultivos y Aprovechamientos de la provincia (MAPA, 1986). No obstante, como prácticamente la repoblación privada es nula, estos montes reflejan la totalidad de la superficie repoblada en la provincia, por lo que su representatividad es completa en lo que al proceso se refiere.

Dada la distribución de pino carrasco en la provincia y las regiones que se pueden definir al analizar las formaciones naturales de esta especie en el país (NICOLÁS \& GANDULLO, 1972), Alicante presenta una variabilidad de ambientes que asegura un gradiente completo de las variaciones del clima mediterráneo (PASTOR, 1992). Todos los subambientes descritos en el mapa geocientífico de la provincia (A.M.A., 1987), presentan extensiones de repoblación forestal. La variabilidad comentada se refleja también desde el punto de vista de la productividad potencial para los ecosistemas de la zona, así GANDULLO \& MUÑOZ (1987) establecen la existencia de 8 de los 9 niveles de producción que definen.

\section{MÉTODOS}

La información presentada se elabora a partir del balance de actividades sobre los montes gestionados por la Unidad Forestal (antigua dirección provincial del ICONA) y de un muestreo de 120 estaciones (Fig. $\mathrm{n}^{\circ}$ 1), definidas dentro de dichos montes que se realizó entre 1985 y 1986.

El archivo de actividades consiste en una recopilación en fichas por montes, según el catálogo de la provincia, que refleja cualquier actividad desempeñada, indicándose el año y la superficie afectada. Este archivo permite definir cuánto se repobló y en qué año, o cuánta superficie fue podada en dicho monte.

La existencia de cartografía de escala adecuada (E 1:10.000 o mayor) no parece haber estado disponible en la mayoría de los casos, lo que ha reducido los registros de muchas actuaciones a la memoria y anotaciones de los agentes forestales. Esta circunstancia nos condujo a la necesaria realización de un muestreo que fuera representativo de la diversidad presente en los diferentes montes. Debido a que nuestro objetivo se centraba principalmente en la explicación de la productividad, el criterio seguido para la selección de las parcelas dentro de cada monte catalogado fue muestrear las zonas que mejor y que peor desarrollos presentaban. En algunos casos, donde la superficie era considerable, se elegían además otras parcelas que fueran representativas de las condiciones medias o de las más frecuentes para dicho enclave. 


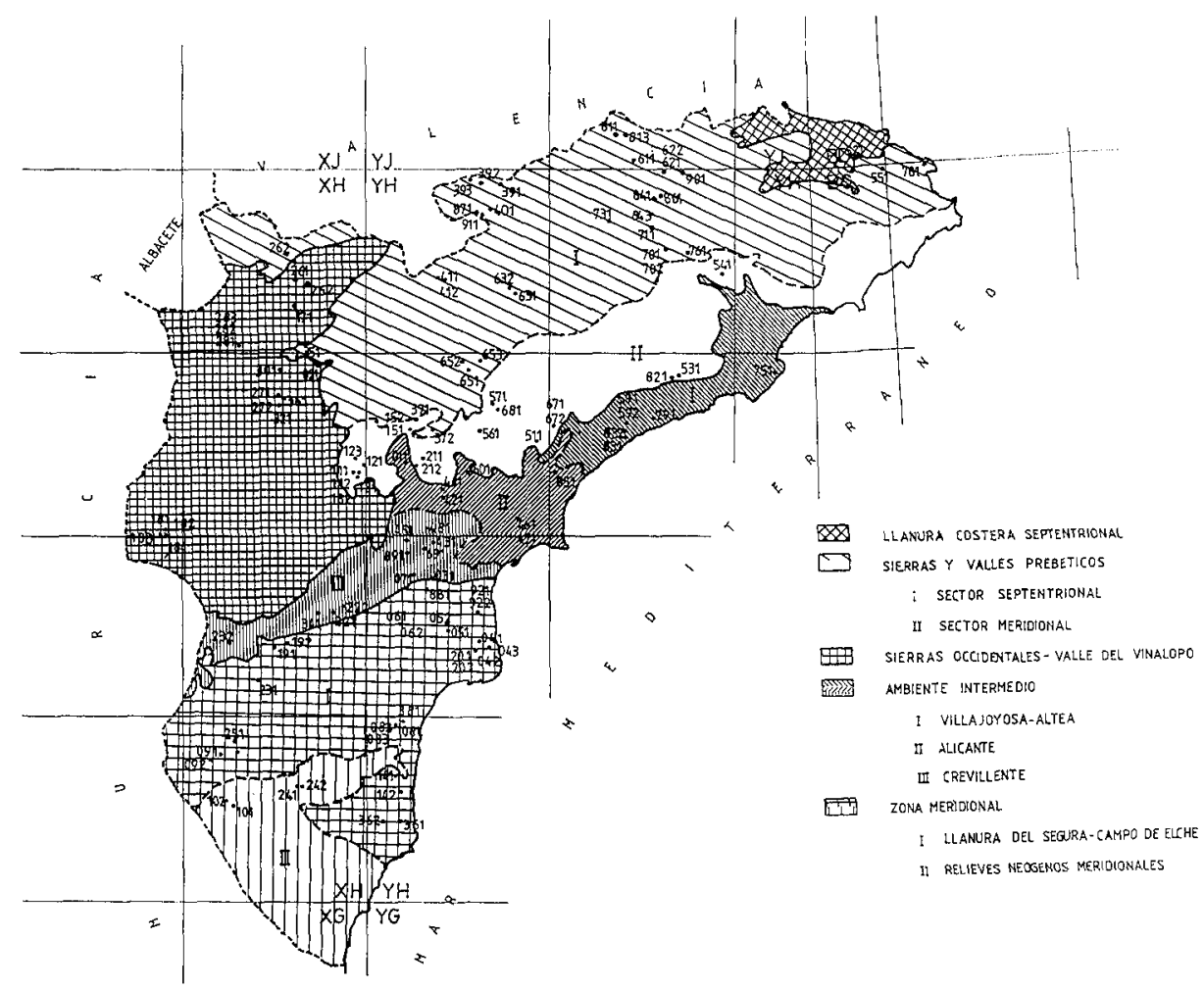

Fig. $n^{2}$ 1.-Localización de las repoblaciones muestreadas en la provincia de Alicante.

En cada una de las estaciones se recopilaron variables ambientales que caracterizan el enclave desde una perspectiva fisiográfica, litológica, edáfica y climática; variables culturales que reflejan los procedimientos seguidos para la realización de las plantaciones y variables bióticas que expresan las características del pino carrasco que se desarrolla en dichas condiciones.

Las variables consideradas en cada grupo así como el método de su obtención fue:

fisiográficas: (altitud) por lectura del mapa topográfico; (pendiente) determinada mediante un clinómetro; (orientación) se obtiene por asignación a uno de los 16 sectores definidos más las zonas llanas.

litológicas: se determinaron empleando los mapas geológicos del I.G.M.E. E1: 50000 serie 1972. La diversidad de tipos existentes se agrupó en 10 clases según sus similitudes geoquímicas y la trayectoria en cuanto a la formación de suelo. Las clases son: Calizas (CA); Calizas tableadas (CT); Calizas y Margas (CM); Dolomías (DO); Caliche (CL); Margas (MA); Margas con yesos (MY); Areniscas Calcáreas (AC); Detríticos sueltos (DS) y Arenas (AR). 
edáficas: se han efectuado los análisis siguientes para caracterizar los suelos: textura, materiargánica, carbonatos, fósforo extractable total, nitrógeno total, $\mathrm{pH}$ en agua, $\mathrm{pH}$ en $\mathrm{Cl} 2 \mathrm{Ca}$.

climáticas: se seleccionaron 33 estaciones meteorológicas, 23 termopluviométricas y 10 pluviométricas, de las cuales se obtuvieron series de 30 años para la caracterización de las diferentes parcelas de muestreo. La asignación a cada parcela de las variables de precipitación y temperatura se realizó por proximidad y por pertenecer al mismo intervalo en el mapa provincial de isoyetas e isotermas. Las variables empleadas para la presente caracterización se fundamentan en los registros a nivel mensual para los distintos observatorios.

Se siguió la clasificación definida por NAHAL (1981), que fue adoptada por SPECHT (1988) para la caracterización de todas las distintas zonas con climas mediterráneos del mundo.

\section{TABLA 1}

\begin{tabular}{lrrrrrl}
\hline CONDICIÓN & $\mathbf{P}$ & $\mathbf{T}$ & $\mathbf{M}$ & $\mathbf{m}$ & $\mathbf{Q}$ & estación \\
\hline AR-C & 299 & 17.8 & 31.3 & 6.8 & 41.8 & Guardamar \\
AR-T & 294 & 17.5 & 34.0 & 4.2 & 33.7 & Catral \\
SAR-C & 302 & 18.2 & 30.6 & 7.7 & 45.0 & Orihuela \\
SAR-FI & 365 & 14.1 & 31.4 & -0.5 & 40.2 & Villena \\
SU-C & 556 & 16.4 & 28.9 & 7.1 & 87.4 & Callosa E. \\
SU-T & 905 & 17.3 & 30.6 & 4.5 & 119.0 & Pego \\
\hline
\end{tabular}

Tabla $n^{2}$ 1.-Descriptores climáticos que definen el rango de condiciones en la provincia de Alicante.

Condición: $\mathrm{AR}=$ árido; $\mathrm{SAR}=$ semiárido; $\mathrm{SU}=$ subhúmedo;

$\mathrm{C}=$ cálido; $\mathrm{T}=$ templado; $\mathrm{FI}=$ frío;

Variables: $\mathrm{P}=$ precipitación media anual $(\mathrm{mm}.) ; \mathrm{T}=$ media mensual de las temperaturas medias; $\mathrm{M}=$ media mensual de las temperaturas medias; $\mathrm{m}=$ media mensual de las temperaturas mínimas.

Temperatura en ${ }^{\circ}$ Celsius; $\mathrm{Q}=$ Cociente pluviotérmico de Emberger.

Para la caracterización de las masas de pino carrasco se realizaron mediciones en parcelas de más de $200 \mathrm{~m}^{2}$ y que contuvieran 30 pies como mínimo. Dada su homogeneidad en el marco y momento de plantación, la variabilidad individual se esperaba que fuera menor que en condiciones de regeneración natural y sin preparación del terreno (tal como indicaba BOUCHON (1975), la altura media reflejaba bien la características de la altura dominante y por ello podía emplearse en la asignación de los índices de calidad).

Las medidas relativas a la vegetación preexistente no se conocen dada la carencia de ficheros con esta información. La edad relativamente temprana de las repoblaciones asegura que la vegetación previa no ha desaparecido en muchos casos del sotobosque. Por este motivo y con el objeto de tener una visión general de las comunidades vegetales que iban a estar afectadas por el proceso de repobla- 
ción, se realizó la caracterización respecto a coberturas de las especies dominantes en el sotobosque. Esta información indicaría superficialmente las condiciones en que se encontraba todo el sistema respecto a su complejidad estructural, hasta qué punto esta vegetación constituye un compartimento importante del sistema y si el sistema en sí todavía guarda una buena capacidad regenerativa a nivel del conjunto de la cubierta vegetal ante una perturbación que destruyera por completo toda la masa de Pinus halepensis. Se recopiló la información de las especies dominantes en el momento del muestreo dando un valor de cobertura visual que indicaba.

La información sobre los aspectos relativos a la implantación y mantenimiento de las masas fueron recopilados "in situ" por observación directa. En lo que se refiere a los métodos de plantación, los tres tipos son identificables incluso en las estaciones de mayor calidad y edad. Respecto a los tratamientos selvícolas postestablecimiento, tan sólo las podas podían identificarse. El número de veces se podía deducir por el aspecto de los tocones, aunque su datación requiere un análisis por sección. La caracterización de la supervivencia postplantación se puede comprobar en algunas zonas de más edad porque se conservan los hoyos vacíos que se realizaron para la implantación. Sin embargo, dada la "reposición de marras" en un período de 1 a 5 años después de la plantación original con nuevos plantones, hace que en ocasiones estos huecos representan una mortalidad posterior, motivada por plagas (ej. Blastophagus sp. (barrenadores) o por defoliadores Thaumetopoea pithyocampa).

A fin de poder determinar la calidad y respuesta de la especie, los árboles incluidos en la parcela eran medidos para su caracterización dasométrica. Las variables consideradas son: Perímetro del tronco en la base, Perímetro a 0.5 y a $1.3 \mathrm{~m}$., siempre que fuera posible, Altura total de cada individuo, obtenida mediante el empleo de una pértiga y excepcionalmente en los ejemplares de mayor tamaño mediante el uso de un clinómetro, Densidad de la masa calculada a partir de la superficie y del número de árboles existentes en la parcela muestreada.

\section{RESULTADOS Y DISCUSIÓN}

Para presentar las características cuantificadas de los descriptores abordamos este apartado con la información sobre cuándo, cómo y dónde se repobló, pretendiendo mostrar con claridad la evolución del proceso dada la información disponible.

\section{Cuándo se repuebla. Balance de actividades 1940-1984.}

La evolución anual tanto de la superficie repoblada como del número de actuaciones se muestra en la fig. $\mathrm{n}^{\mathrm{O}} 2$. Destaca una estructura bimodal que presenta un máximo de 2.200 ha. de superficie repoblada en 1955 y un amplio período desde 1964 a 1984 sin una tendencia clara, donde la superficie anual repoblada oscila entre 500 y 1.000 ha. Desde 1940, la actividad es claramente creciente hasta llegar a 1955 y coincide con un período posterior a la guerra civil, donde la repo- 
blación forestal aparece como un forma de contratación de jornaleros (GROOME, H.J., 1990), dada la difícil situación económica y la dificultad de las labores de preparación del terreno por la carencia de maquinaria. Entre 1956 y 1963 se produce una considerable caída que con la excepción de 1957, en que se repueblan prácticamente 900 ha., para el resto del período los valores oscilan entre 100 y 300 ha/año. A partir del año 1978 se observa una disminución considerable de la superficie repoblada.

Con posterioridad a este año se produce un fenómeno de sustitución de la actividad repobladora por tratamientos selvícolas (podas, cortafuegos...).

Paradójicamente la tendencia es opuesta a la que señala GARCÍA SALMERÓN (1991) para el resto del Estado, donde el pino carrasco aparece como la primera especie empleada en repoblaciones en lo que a superficie se refiere durante el sexenio 1978-1983.

El número de actuaciones se solapa, mostrando que no hay una gran variación entre los tamaños de las diferentes zonas repobladas. Este nunca ha superado las 35 anuales, siendo normales de 10 a 20 actuaciones antes de 1955 , y de 8 a 15 con posterioridad al 1965. La superficie de cada actuación, oscila entre 43 y 133 ha. y refleja una tendencia decreciente con el tiempo. A diferencia de otras provincias, la especie empleada ha sido casi con total exclusividad Pinus halepensis.

En lo referente a la propiedad de los terrenos sobre los que se actúa, es curioso observar que desde 1950 la superficie consorciada ha superado siempre, a

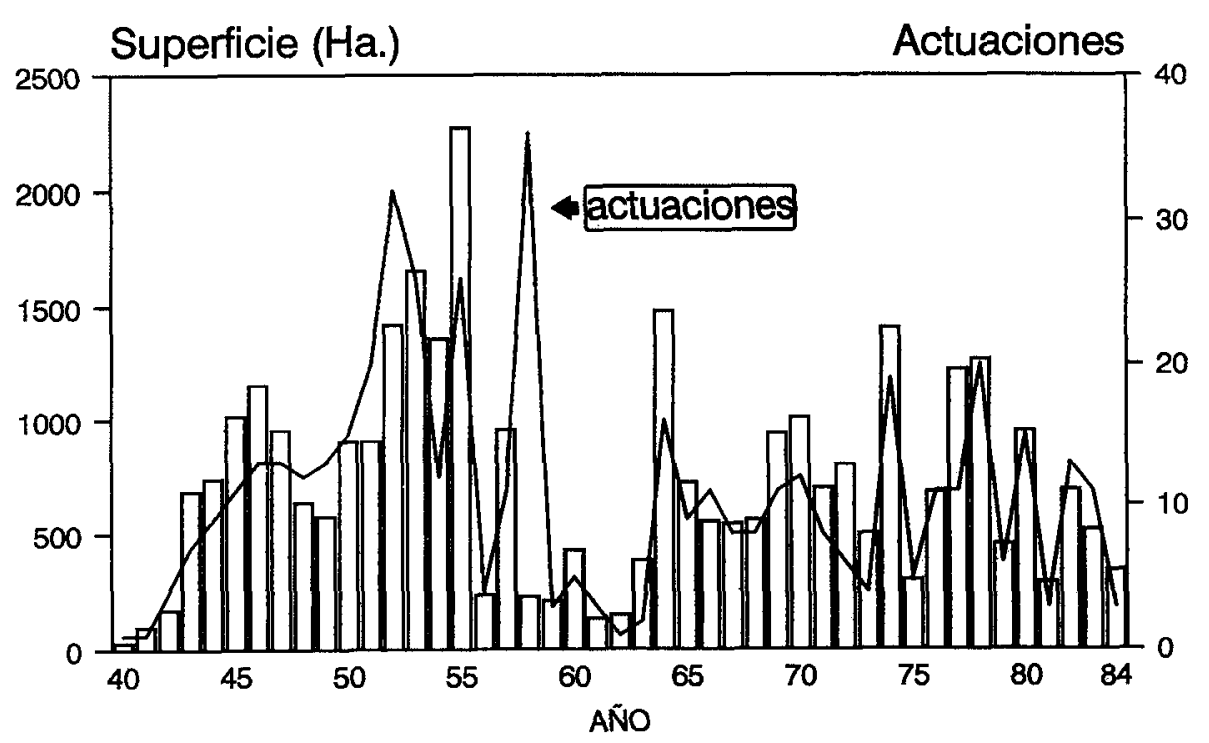

Fig. $n^{\circ} 2$.- Superficie repoblada y número de actuaciones. 
excepción de en 1977, a la estatal. Este es el único año en que la superficie estatal repoblada supera las 1000 ha. El máximo absoluto de repoblación se consigue claramente por la repoblación de 2.000 ha. consorciadas en 1955. Básicamente este hecho refleja la realidad respecto a la propiedad de los montes o de la superficie forestal en la provincia. Las implicaciones que dicha situación puede tener en un futuro sobre la gestión de dichas superficies es importante y requiere una mayor consideración, dada la naturaleza protectora de prácticamente la totalidad de las masas.

En base a la información del Mapa de Cultivos y Aprovechamientos de la provincia, la superficie forestal representa un $39.9 \%$ de ella, lo que equivale a 232.454 ha. Ese porcentaje lo constituyen 4 tipos de vegetación natural, aportando los pastos un $0.09 \%$, el matorral arbolado un $5.7 \%$, las formaciones arbóreas un $12.5 \%$ y el matorral no arbolado el $21.6 \%$. La superficie repoblada en la provincia desde 1940 ha sido de 33.241 ha., lo que representa un 5.7\%, siendo la extensión de pino carrasco repoblada preexistente de $41.583 \mathrm{ha}$.

La ausencia de una cartografía histórica de los incendios forestales hasta prácticamente 1972 hace que la elaboración de un balance preciso de la superficie existente de pino carrasco no sea una tarea fácil. La superficie quemada entre 1972 y 1991 en incendios de más de 100 hectáreas, alcanza las 62.074 ha. No es posible discernir, a partir de los registros existentes, qué porcentaje de esta superficie es de repoblaciones, no obstante es evidente que la superficie supera considerablemente la repoblada desde 1940. Los incendios han afectado en alguna ocasión al 48,6\% de los términos municipales y en estos, la superficie quemada durante los 20 años considerados podría alcanzar un $55,8 \%$ de la vegetación natural.

\section{Características de la muestra de repoblaciones.}

En el histograma de edad de las 120 estaciones muestreadas (Fig. $\mathrm{n}^{\circ}$ 3), se observa con bastante precisión que es bimodal y que refleja la evolución del proceso repoblador general. El valor medio de la edad de la muestra es de 21 años y el rango va de 3 a 42 años. La mayor frecuencia se encuentra a los 11 años. En general, parece estar más representado el período comprendido entre 1965 y 1984.

Otras informaciones que serían interesantes de conocer pero que los ficheros no las recopilan son:

1) La determinación precisa de las fechas de plantación. Esta información sirve para valorar los niveles de mortalidad post-plantación, y definir los regímenes de precipitación y temperatura más beneficiosos.

2) El seguimiento cronológico de las perturbaciones a las que se ven sometidas. En la provincia son de cuatro tipos principalmente: Fuego predominantemente en el sector norte, pudiendo extenderse hasta una línea imaginaria entre Alicante y Villena. Defoliadores como la procesionaria Thaumetopoea pityocampa que afecta a prácticamente todas las zonas repobladas, pero es en las zonas con mayo- 


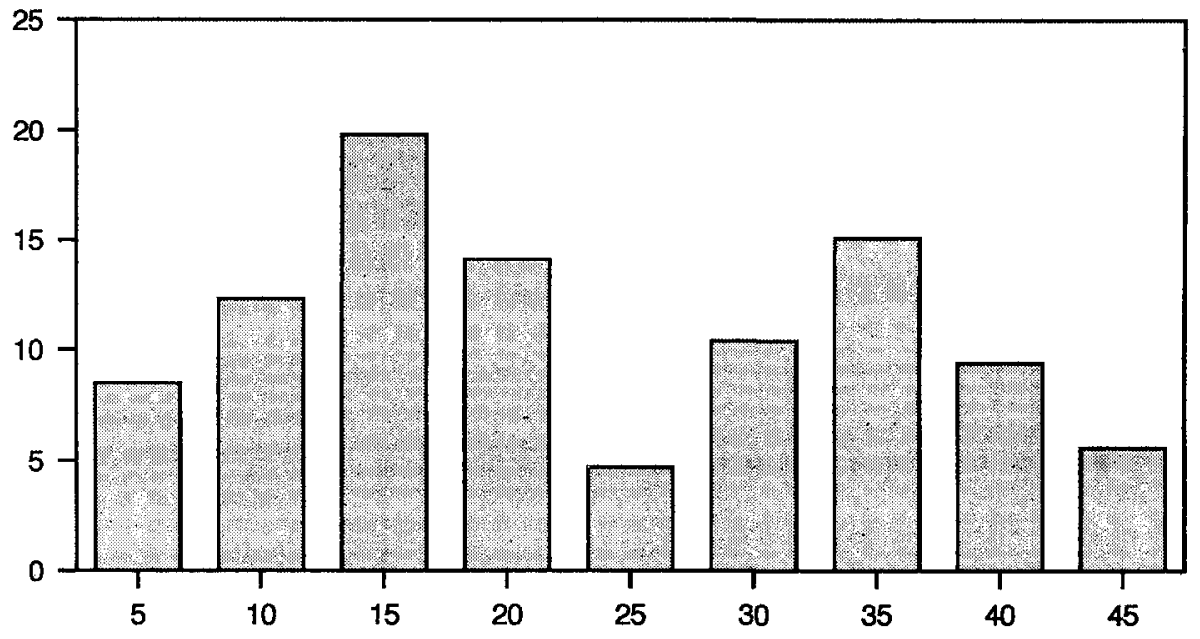

Fig. $n^{2} 3$. -Edades de las repoblaciones. (Frecuencias relativas)

res stress hídrico donde su efecto negativo es más considerable. Ataques fúngicos; se han observado en zonas puntuales de la provincia clorosis agudas que provocaban la muerte de las acículas aunque no su desfronde. Como se comenta en PASTOR (1992), estos procesos en los que no está claramente definida todavía la causa, constituyen una importante modificación en el régimen de ciclado de materia orgánica. Aunque inicialmente y en un corto período de tiempo tras su aparición se produce un incremento momentáneo del desfronde, con posterioridad éste se ve reducido de forma considerable respecto de su valor potencial. En último lugar, y tal vez como causante de la perturbación anterior, la Contaminación parece estar conectada con la reducción en la productividad de las masas, vía pequeñas necrosis en las acículas.

3) Las características estructurales y florísticas de la vegetación remanente sobre la que se instaura la repoblación es importante de cara a la potencialidad de dicho monocultivo al pasar a ser un sistema de mayor diversidad y complejidad.

4) Otro aspecto que es parte fundamental de cuándo son las características de los plantones, cuántas savias tienen, es decir edad de los mismos.

La importancia que tiene conocer la cronología de los sucesos biológicamente significativos se incrementa por la proyección a medio o largo plazo que toda labor de reforestación conlleva. La recopilación de los efectos provocados por perturbaciones sufridas por una masa repoblada de pino carrasco define las condiciones en las que se produce su crecimiento y es por ello básico para la gestión de la masa. 


\section{Dónde se repuebla}

Por lo general, el área de distribución de una especie refleja el marco de condiciones ecológicas en que ésta es capaz de mantener poblaciones que puedan perpetuarse, lo que requiere que todos los estadios de su ciclo vegetativo puedan completarse adecuadamente en el marco de las interacciones físicas y biológicas que definen su hábitat.

La repoblación forestal introduce nuevos individuos en zonas en las que teóricamente podían crecer, como un mecanismo de acelerar la colonización, incrementando artificialmente la presencia y densidad de plantones. Su existencia indica la viabilidad de la especie para crecer en dicha zona, aunque sólo la evidencia de que la especie se reproduce adecuadamente, sugiere que las condiciones coinciden ecológicamente con las típicas de su área de distribución natural.

En la provincia de Alicante, se introduce pino carrasco en dos tipos de ambientes: a) los que no presentan evidencias de haber mantenido una población natural de la especie en un período inferior a 50 años. Estos son frecuentes en la mayor parte de las zonas semiáridas y áridas de la provincia. b) los de las zonas en las que, dada la frecuencia de incendios, han perdido la cobertura de masas de pino carrasco y donde el sotobosque presentaba un buen desarrollo, pasando éste a constituir una vegetación con cobertura superior al $50 \%$ en la actualidad. Estas zonas se corresponden con la mayoría del sector subhúmedo, que se extiende predominantemente del Centro al Norte de la provincia.

Una aproximación de las condiciones macroclimáticas en que se encuentran las zonas repobladas nos indica que la zona con mayor superficie es la subhúmeda, seguida de la semiárida cálida y de la semiárida fría. Esta información superficial no revela una mayor idoneidad sino que, en una buena parte de los casos, hay una tendencia motivada exclusivamente por la disponibilidad de terrenos para repoblación. En cualquier caso, refleja la situación actual.

La caracterización fisiográfica de las repoblaciones da una idea de las condiciones microambientales en las que se encuentran. El rango de altitud oscila entre 10 y 1.120 metros; el $52 \%$ aparece por debajo de los $420 \mathrm{~m}$. y un $25 \%$ de los casos superan los $700 \mathrm{~m}$. Respecto a la pendiente, el $50 \%$ de las estaciones presentan una inclinación inferior a 7.6 grados aunque en situaciones excepcionales puede llegarse a los 35 grados. Los valores 2.5 y 12.5 grados representan los límites que dividirían la muestra en 3 grupos igualmente representados. La orientación de las parcelas se caracterizó en 16 sectores. Las parcelas llanas representan la mayor frecuencia con $13 \%$ de las estaciones, y las menos representadas son NEE, SOO y ONO. Con valores próximos al $10 \%$ se encuentran E, SE y NO, presentando el resto valores alrededor de un $4 \%$. En general, si consideramos las zonas llanas incluidas en la categoría de solana, el $59.7 \%$ de las estaciones pertenecerían a este grupo siendo las umbrías un $40.3 \%$ 
Un aspecto fundamental esta representado por el tipo de sustratos donde se repuebla (Tabla $\mathrm{n}^{\circ} 2$ ). La descripción indica que los sustratos desarrollados sobre rocas madres del tipo de caliches o costras calcáreas son los que dominan con un $20 \%$ aproximadamente. Los de menor frecuencia son las arenas y las rocas dolomíticas. Cuatro tipos de rocas: Calizas, Calizas y margas, Margas con yesos y Detritos sueltos, con frecuencias individuales de alrededor del $12 \%$, representan otro bloque. Calizas tableadas, Margas y Areniscas calcáreas presentan valores individuales del 7 al 10\%. La roca madre es importante en la definición de la calidad de la masa de pino carrasco según SCHILLER (1982).

La tabla $n^{\circ} 2$ resalta en primer lugar una marcada diferencia entre las zonas climáticas en las que hay repoblaciones. La mayor frecuencia se encuentra en el semiárido con un $58 \%$ de los casos, siendo los valores para árido y subhúmedo idénticos con un $21 \%$ respectivamente. A nivel de los grupos de edad, la representación es mucho más homogénea con una representación del 33.9, 36.4, 29.7 por ciento, considerando los intervalos en orden de edad creciente.

Con el objeto de determinar si existían tendencias en la repoblación dentro de cada zona climática, se han calculado los porcentajes relativos de cada roca madre en función de los casos existentes para cada una de ellas. Se observa que existe una tendencia a que las repoblaciones en clima árido se concentren en menor número de tipos de roca madre, mientras que en semiárido y subhúmedo los valores son más homogéneos. A excepción del tipo Detritos sueltos, donde existen porcentajes próximos en las tres condiciones climáticas, no se observa una tendencia a

repoblar determinados tipos de roca madre. En el árido, predominan Caliches y Areniscas calcáreas, con Margas y Detritos sueltos en una posición intermedia. En el semiárido, aunque la mayor frecuencia se encuentra en Margas con yesos, las Calizas, Calizas margosas, Caliches y Detritos sueltos, presentan valores prácticamente equivalentes. En el subhúmedo, Calizas margosas dominan pero Calizas, Calizas tableadas, Dolomías, Caliches y Detritos sueltos ocupan una posición intermedia.

Entre 1971 y 1985 se repobló predominantemente sobre Calizas margosas y Caliches. Sobre Caliches entre 1956 y 1970 y sobre Calizas y Detritos sueltos con anterioridad a 1956.

Tal como JENNY (1980) señala, las características de un suelo están definidas directamente por las condiciones climáticas, la naturaleza de la roca madre, la topografía, la actividad de organismos y el tiempo transcurrido desde que un determinado sustrato se encontraba expuesto a estos factores. A escala temporal humana estos procesos se pueden considerar poco frecuentes y muy localizados en el espacio. Son más frecuentes perturbaciones que modifican parcialmente las condiciones del sustrato existente como pueden ser fenómenos erosivos o actividades motivadas por el hombre. 
TABLA 2

FRECUENCIAS RELATIVAS DE REPOBLACIÓN

POR TIPOS DE ROCA MADRE

\begin{tabular}{|c|c|c|c|c|c|c|}
\hline & & CLIMA & & & EDAD & \\
\hline $\begin{array}{l}\text { ROCA } \\
\text { MADRE }\end{array}$ & $\mathrm{AR}$ & SAR & SUH & $<15$ & $\begin{array}{l}15 \mathrm{a} \\
29\end{array}$ & $<29$ \\
\hline \multirow{2}{*}{ CALIZAS } & 0 & 9.3 & 3.4 & 3.4 & 3.4 & 5.9 \\
\hline & 0 & 16.1 & 16.0 & 10.0 & 9.3 & 20.0 \\
\hline \multirow{2}{*}{$\begin{array}{l}\text { CALIZAS } \\
\text { TABLEADAS }\end{array}$} & 0.8 & 2.5 & 3.4 & 4.2 & 1.7 & 0.8 \\
\hline & 4 & 4.4 & 16.0 & 12.5 & 4.6 & 2.9 \\
\hline \multirow{2}{*}{$\begin{array}{l}\text { CALIZAS Y } \\
\text { MARGAS }\end{array}$} & 0.8 & 9.3 & 4.2 & 7.6 & 5.1 & 1.7 \\
\hline & 4 & 16.1 & 20.0 & 22.5 & 13.9 & 5.7 \\
\hline \multirow{2}{*}{ DOLOMIAS } & 0 & 1.7 & 2.5 & 1.7 & 0 & 2.5 \\
\hline & 0 & 2.9 & 12.0 & 5 & 0 & 8.6 \\
\hline \multirow{2}{*}{ CALICHE } & 6.8 & 9.3 & 2.5 & 5.9 & 8.5 & 4.2 \\
\hline & 32.0 & 16.1 & 12.0 & 17.5 & 23.3 & 14.3 \\
\hline \multirow{2}{*}{ MARGAS } & 3.4 & 5.1 & 0.8 & 3.4 & 3.4 & 2.5 \\
\hline & 16.0 & 8.8 & 4 & 10.0 & 9.3 & 8.6 \\
\hline \multirow{2}{*}{$\begin{array}{l}\text { MARGAS Y } \\
\text { YESOS }\end{array}$} & 0.8 & 10.2 & 1.7 & 3.4 & 5.1 & 4.2 \\
\hline & 4 & 17.6 & 8 & 10.0 & 13.9 & 14.3 \\
\hline \multirow{2}{*}{ ARENAS } & 0.8 & 0 & 0 & 0 & 0.8 & 0 \\
\hline & 4 & 0 & 0 & 0 & 2.3 & 0 \\
\hline \multirow{2}{*}{$\begin{array}{l}\text { DETRITOS } \\
\text { SUELTOS }\end{array}$} & 3.4 & 7.6 & 2.5 & 3.4 & 4.2 & 5.9 \\
\hline & 16.0 & 13.2 & 12.0 & 10.0 & 11.6 & 20.0 \\
\hline \multirow{2}{*}{$\begin{array}{l}\text { ARENISCA } \\
\text { CALCÁREA }\end{array}$} & 4.2 & 1.7 & 0 & 0 & 4.2 & 1.7 \\
\hline & 20.0 & 2.9 & 0 & 0 & 11.6 & 5.7 \\
\hline
\end{tabular}

Tabla $\mathrm{n}^{2}$ 2.-Frecuencias relativas de repoblaciones en distintos tipos de roca madre según clases climáticas y de edad. Se representan las frecuencias relativas respecto al total de casos estudiados y respecto al total de casos en la clase de clima o edad considerada. 
Las labores de preparación del terreno en repoblaciones constituyen una considerable perturbación del estado natural de dichos suelos o sustratos. Esta actividad puede o no afectar a la roca madre dependiendo normalmente del volumen de sustrato disponible en los horizontes superiores. En determinadas circunstancias donde aparecen costras calcáreas o calízas karstificadas con restos de terra rossa en las cavidades, la preparación aumenta la cantidad de suelo o la accesibilidad de las raíces al mismo. Las diferencias que la preparación del terreno genera no se han comparado con el suelo natural. Por otra parte, la acción de la repoblación puede modificar las características y no existe bibliografía que defina cuáles son los cambios que se producen.

La caracterización de las variables químicas del suelo consideradas se incluyen en otro artículo PASTOR-LÓPEZ \& MARTÍN (1994), en el que se discute la influencia que este hecho puede tener en las mismas. SCHILLER (1982) recalca que la dureza de la roca es el factor que mayor influencia tiene en el desarrollo de pino carrasco en Israel, ya que esta propiedad afecta a la disponibilidad de agua y a la estructura química que adoptan determinados nutrientes como el nitrógeno. Concluye que las formaciones litológicas que contienen margas y cretas constituyen los mejores sitios para el pino carrasco y que por lo tanto deberían de ser los lugares prioritarios a repoblar. Aunque no se ha valorado todavía estadísticamente si tal relación se cumple en las masas de la provincia de Alicante, o si la roca madre requiere más atención en cuanto a su definición, los sustratos del tipo margas o "calizas y margas" representan cerca de un $25 \%$ de las condiciones observadas, no obstante aproximadamente un $32 \%$ de las rocas madres estudiadas (margas y yesos, detritos sueltos, areniscas areniscas calcáreas y arenas) no fueron consideradas en la investigación de SCHILLER (1982).

La caracterización textural de los suelos descritos presenta una concentración del conjunto de suelos en 3 tipos que contrastan con la variabilidad de rocas madres comentada. Los suelos con textura franco-limosa dominan representando algo más de un $50 \%$ de los casos. Las texturas franco-arenosa y franca alcanzan 21 y $19 \%$ de las estaciones respectivamente. Las restantes texturas están con representación individual inferior al $2 \%$ y son, por orden de importancia: franco-arenosa, franco-arcillosa, arenoso-franca, arenosa y arcillosa.

Otro aspecto de singular importancia son las características de la vegetación existente. Dada la variabilidad en edad y zona climática, las combinaciones de estos dos factores se emplean para su caracterización. En la tabla no 3 se presentan los rangos de cobertura total arbustiva para las condiciones de edad y clima. 


\begin{tabular}{cccc}
\hline EDAD & Árido & $\begin{array}{c}\text { CLIMA } \\
\text { Semiárido }\end{array}$ & Subhúmedo \\
\hline$<15$ & $40 / 15 / 3$ & $80 / 2 / 20$ & $90 / 2 / 17$ \\
15 a 29 & $40 / 2 / 20$ & $100 / 2 / 23$ & $-/-/ 0$ \\
$>29$ & $10 / 5 / 2$ & $60 / 2 / 25$ & $90 / 2 / 8$ \\
\hline
\end{tabular}

Tabla $n^{2}$ 3. - Coberturas porcentuales del sotobosque. La $1^{\mathrm{a}}$ cifra corresponde a la cobertura total arbustiva y herbácea máxima, y la $2^{\underline{a}}$ a la cobertura mínima. La tercera cifra representa el número de estaciones del grupo.

Las especies arbustivas que presentan una mayor cobertura en el área subhúmeda son Ulex parviflorus, Quercus coccifera y Rosmarinus officinalis, estas dos últimas son asimismo las más frecuentes en la zona semiárida junto con Erica multiflora y Globularia alypum. En la zona árida hay todo un grupo de especies de Tymus, Teucrium, Fumana, Sideritis etc., sin que ninguna de ellas llegue a ser dominante. El estrato herbáceo está dominado por Brachypodium retussum en los tres climas y en las zonas semiáridas; de forma puntual se presenta combinado con Stipa tenacissima. NICOLÁS \& GANDULLO (1972) incluyen la provincia de Alicante en las zonas que denominan Levante y Sudeste. En ambas regiones Rosmarinus officinalis, Quercus coccifera y Juniperus oxycedrus son las tres especies que presentan un mayor índice regional de frecuencia. Estas especies son teóricamente características de lo que se define como masas naturales de pino carrasco.

\section{Cómo se repuebla}

Básicamente cualquier repoblación sigue cuatro etapas que la caracterizan: 1) Preparación del terreno, 2) Producción de plantones, 3) Plantación, y 4) Manejo postplantación. La información disponible sobre cada uno de estos aspectos es completamente variable en la realidad de la provincia de Alicante y del Estado. Fundamentalmente está relacionada con los recursos de interés económico de los distintos tipos de masas. A este respecto, parece evidente que se ha prestado una atención casi exclusiva al desarrollo de la especie implantada, careciéndose por lo general de información relativa a los efectos de los componentes físicos y biológicos implicados. Esta visión aproxima considerablemente las repoblaciones a labores agrícolas, lo que resulta muy peligroso sobre todo en zonas donde los objetivos son la creación de bosques protectores.

En estas circunstancias, la propiedad de autosuficiencia y autoperpetuación de los sistemas creados (que carece de sentido en la mayoría de sistemas agrícolas), 
es esencial para establecer los aspectos que deben de preocupar a la hora de definir los criterios justificativos una acción repobladora. La transferencia de procedimientos desarrollados y experimentados para otro tipo de ecosistemas se ha demostrado muy negativa en distintas áreas de nuestro país. A pesar de la necesidad de desarrollar una selvicultura mediterránea, su puesta a punto es prácticamente un ejercicio literario, y la escasez en general de actividades de revegetación en nuestro país dificulta vías alternativas a las ligadas directamente con las instituciones administrativas. Sería importante en la línea de las sugerencias realizadas por instituciones como la del Madison Arboretum (JORDAN et al., 1982) que la ecología de la restauración se proyecte en todos los frentes posibles como una nueva ética de actuación frente a la gestión de la cubierta vegetal.

\section{Preparación del terreno.}

Se entiende por este término al conjunto de acciones que se llevan a cabo sobre una zona para asegurar el éxito en la implantación y posterior desarrollo de la masa forestal. Los objetivos principales los define GARCÍA SALMERÓN (1991), y de forma más concreta DE SIMON (1990) proponiendo como objetivos para zonas áridas: 1) recolectar el agua de una determinada superficie o zona de impluvio y dirigirla hacia el área receptora donde se repuebla (cosecha de agua); 2) mejorar la capacidad de almacenamiento y de infiltración en el área de plantación (preparación del suelo); 3) ordenar el drenaje natural y desaguar las escorrentías en los drenes naturales (sistematización del suelo).

En realidad, como indican CUBBAGE et al. (1991), los métodos de preparación del terreno son una de las acciones más costosas a abordar. Por otra parte, han resultado ser una de las causas de mayor conflictividad social de los procesos de reforestación en nuestro país. Representan un riesgo de perturbación considerable porque en muchas ocasiones su empleo no se ha ponderado más que como una forma efectiva de aumentar considerablemente la capacidad repobladora en superficie. El equilibrio en el empleo positivo de estas tecnologías y la conservación de la estructura de los suelos y vegetación preexistente no se ha analizado cuantitativamente. No obstante, su desarrollo resulta fundamental a la hora de abordar uno de los aspectos cruciales de la tan pretendida silvicultura mediterránea.

Los procesos considerados dentro de la preparación del terreno pueden abarcar tal como recoge DUREYA \& DOUGHERTY (1991) acciones como los fuegos prescritos (prescribed fires), así como la preparación química tanto con herbicidas como con fertilizantes. Estos métodos no se han empleado en la provincia, aunque no debería descartarse sobre todo el primero en lo que se refiere a la gestión de las zonas con alta frecuencia de incendios del norte de la provincia, donde las masas de pino carrasco han sido claramente desplazadas.

SHEPHERD (1986) señala que existe una considerable diferencia en el tipo de acciones involucradas en función de dos condicionantes: 1) que se esté repoblando una zona que no sostenía bosques previamente (en un período de 50 años) 
o donde si existían previamente y la acción es una sustitución de la masa previa; y 2) de la pendiente del terreno.

Los métodos empleados durante el período de repoblación han sido 3 predominantemente: repoblación manual con hoyos, subsolado o ripping realizado con tractores oruga D-, y terrazas de anchura variable entre 2 y 4 metros. En ocasiones muy localizadas, se encuentra un tipo de repoblación manual denominado fajas, que consiste en la elaboración de terrazas de menos de 0.5 a $1 \mathrm{~m}$. Tan sólo las hemos observado en Jijona, en pendientes de alrededor de $30^{\circ}$ y en suelos de muy poca profundidad. La frecuencia relativa de cada uno de los métodos en el total del período $1940-1985$ es $38 \%, 25 \%$ y $37 \%$. No obstante, el empleo en el tiempo de los diferentes métodos muestra ciertas tendencias claramente, como observamos en la fig. $\mathrm{n}^{\circ} 4$ donde se sugieren dos pautas claras: a) la disminución paulatina desde el quinquenio 1952-56 hasta su desaparición en 1972-76 de los hoyos a mano y b) el aumento creciente desde 1960 tanto de las terrazas como de los surcos o subsolado. Las terrazas son el método de preparación dominante en los años 80 .

\section{Producción de plantones.}

En esta fase se incluyen varios procesos de considerable importancia biológica, como son las características genéticas (procedencia de la semilla) en la producción de la plantula, la selección de los plantones con mayor vigor, la inoculación de micorrizas en los sustratos de germinación (PIOU, 1979). A pesar de su importancia, la información disponible de los orígenes de las semilla es escasa o discontinua a nivel de la Unidad forestal y desconocemos hasta que punto sería posible obtener una información detallada del Instituto de semillas y plantas de vivero del MAPA, que ha sido la institución que ha suministrado habitualmente los lotes de semillas. En lo que se refiere a la germinación y producción del plantón en la

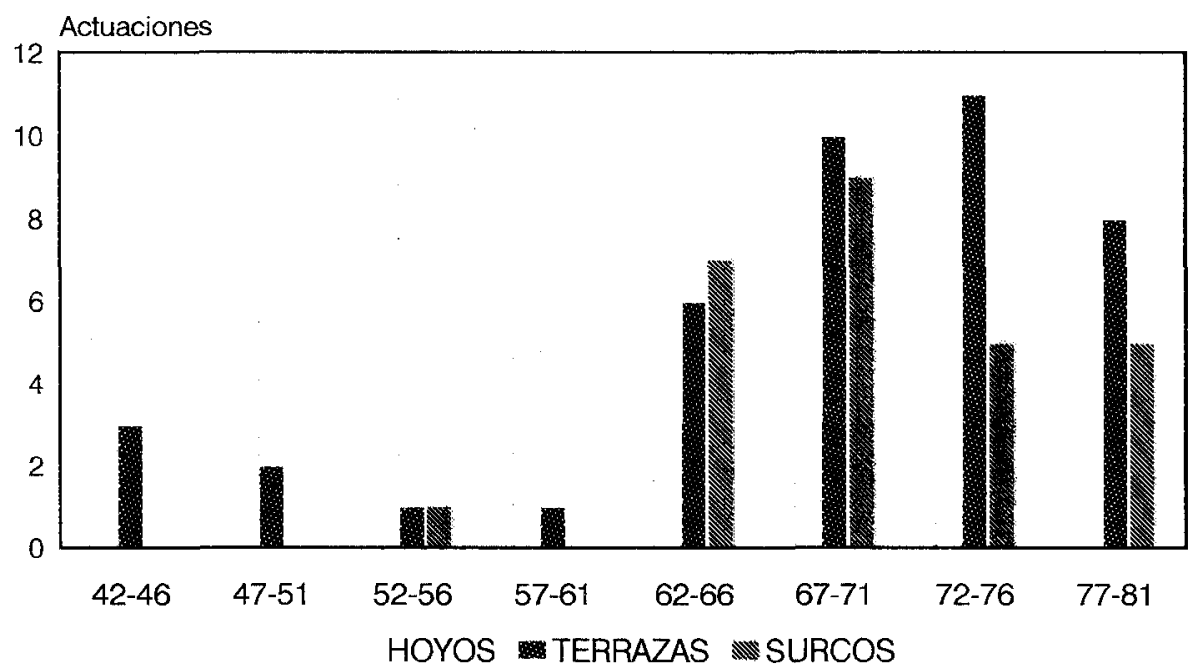

Fig. $n^{2}$ 4.--Preparación del terreno (períodos quincenales). 
mayoría de los casos se han producido en los viveros existentes en Santa Faz y Guardamar del Segura, en el período de tiempo considerado, se ha producido planta para raíz desnuda, en maceta y en bolsa de polietileno. Este último método es el que se emplea en la actualidad. No se han micorrizado en ningún momento dichos plantones.

\section{Plantación.}

La información disponible a este respecto es escasa y aquella que puede estimarse es la que hace referencia a la densidad de plantación. La caracterización de los plantones (parámetros morfológicos, contenidos de agua, vigor, etc) no se ha realizado en absoluto. Estos parámetros han resultado ser fundamentales en los niveles de mortalidad inicial postimplantación para otras especies alternativas en repoblación, BAEZA et al. (1992), aunque el pino carrasco parece ser menos susceptible dada su mayor adaptación a condiciones de xericidad.

El marco de plantación suele ser rectangular o al tresbolillo. El número de pies por hectárea es una característica fundamental de una repoblación y es una de las cuestiones que dificulta la comparación, a nivel de producción, entre localidades durante los estadios juveniles, o en el momento en que existe un equilibrio en el desarrollo entre los pies individuales debido a la competencia. SHEPHERD

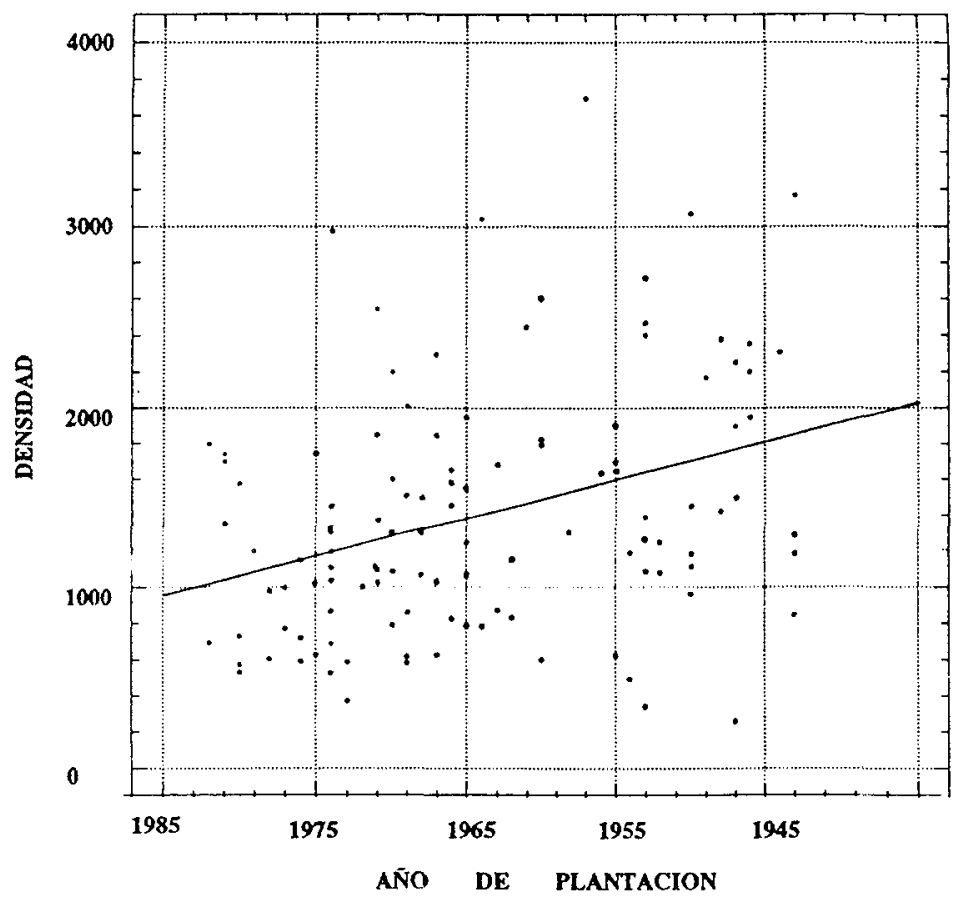

Fig. n 5. - Evolución de la densidad de plantación. 
(1986) indica que la selección de la densidad idónea depende del objetivo a conseguir, ya que tiene un efecto importante tanto en las características del árbol como de la masa.

En nuestra muestra, el rango oscila entre 400 y 3.800 árboles/hectárea. La densidad con una mayor frecuencia es la de 1.200 árboles/hectárea. El 75\% de las estaciones presentan valores inferiores a 1.800 árb./ha. y un 5\% superiores a 3.000 árb./ha. La evolución de la densidad de plantación desde 1940 presenta una tendencia decreciente tal como se observa en la Fig. $\mathrm{n}^{\circ} 5$.

\section{Manejo postplantación.}

Las actividades culturales a las que se somete una repoblación influyen en la fenología y en su tasa de crecimiento. Del análisis de los troncos se puede comprobar sobre la existencia o no de podas. Salvo escasas excepciones, todas las parcelas han sido podadas como mínimo una vez. Esta labor se realiza normalmente alrededor de los cinco años de edad, se conoce como "realce" y consiste en la eliminación del primer verticilo de ramas a fin de favorecer el alargamiento del tronco. La frecuencia de podas posteriores es más irregular, aunque se sitúa entre los 10 y 15 años.

La principal consecuencia de las podas es la inexistencia de necromasa en pie a diferencia de otras masas, así como la modificación de la morfología de toda la planta. Simultáneamente a la poda, suelen realizarse tareas se desbroce, pero su intensidad y extensión resultan difícilmente estandarizables. Estas prácticas se han realizado en ocasiones con fines de prevención contra incendios. Aunque no se han empleado extensivamente dado su coste, representan modificaciones drásticas de todo el sistema.

\section{AGRADECIMIENTOS:}

Esta investigación ha sido subvencionada por el Ministerio de Educación y Ciencia, Consellería de Agricultura y Pesca de la Comunidad Valenciana y Diputación Provincial de Alicante. Los técnicos y agentes forestales de la Unidad Forestal de Alicante tuvieron un papel fundamental en la selección de estaciones y en los muestreos de campo. Queremos expresar nuestro agradecimiento a D. Juan Giner por su apoyo continuado. En particular, queremos agradecer la contribución de Nicolás Giménez, Silvia Ivars y Manuel Valera por su ayuda en las campañas de campo y en la informatización de datos. 


\section{BIBLIOGRAFÍA}

A.M.A.G.V. (1987) Mapa Geocientífico de la provincia de Alicante. E1: 200.000. Tomo I: Memoria. Consultor INGEMISA. Agencia del Medi Ambient, Conselleria d’Administració Publica. pp. 93.

BAEZA et al.

BOUCHON, J. (1974) Les tarifs de cubage. Nancy: E.N.G.R.E.F. Champenoux, INRA. pp. 128

CUBBAGE, F.W.; GUNTER, J.E.; REY, J.; OLSON, T. (1991) Reforestation economics, law and taxation; in: Duryea, M., Dougherty, P. Eds. Forest Generation Manual. Kluwer Academic Pub. Dordrecht. pp.9-31.

DE SIMON, E. (1990) Restauración de la vegetación en cuencas mediterráneas:

Repoblaciones en zonas áridas. Ecología, supl. (1): 401-427.

DURYEA, M.L. DOUGHERTY, P.M. (1991) Forest Regeneration Manual. Kluwer Academic Publishers. Dordrecht; 433.

GARCÍA SALMERÓN, J. (1991) Manual de repoblaciones forestales -1. Fundación Conde del Valle de Salazar. E.T.S. de Ingenieros de Montes. Univ. Politécnica de Madrid; GANDULLO, J.M.; MUÑOZ, L.A. (1987). Mapa de productividad potencial primaria neta de los ecosistemas españoles. Boletín de la Estación Central de Ecología.

JENNY, H. (1980) The Soil Resource. Origin and Behavior. Ecological Studies 37, Springer Verlag. New York. pp. 377.

JORDAN, W.R.; GILPIN, M.E.; ABER, J.D. (1987) Restoration ecology. A synthetic approach to ecological research. Cambridge University Press; pp. M.A.P.A. (1986) Mapa de Cultivos y Aprovechamientos de la provincia de Alicante. E1: 200.000. Memoria. Dirección General de Producción Agraria, M.A.P.A. Madrid. pp. 102.

MUÑOZ, J. (1986) Repoblación forestal. El Campo nº 103; pp 126-128

NAHAL, I. (1981) The Mediterranean climate from a biological viewpoint. in Di Castri, F., D.W. Goodall, R.L. Specht Eds. Mediterranean-type shrublands. Ecosystems of the World. 11. Ch. 3, pp. 63-86. Elsevier Scientific Publishing Company. Amsterdam.

NICOLÁS, A. GANDULLO, J.M. (1972) El estudio ecológico del pino carrasco. Cap. V, en: Ecología de los Pinares Españoles. III. Pinus halepensis M.A.P.A.-I.N.I.A. Madrid. pp. 307.

PASTOR-LÓPEZ (1992) Biomasa, Producción primaria y Flujo de materia orgánica en repoblaciones forestales de la provincia de Alicante. Aplicaciones a la gestión forestal. Tesis Doctoral. Universidad de Alicante. pp. 309.

PIOU, D. (1979) Importance de la mycorrhization dans la resistance au calcaire de divers especes forestieres. Revue Forestiere Française, Vol. 31, $\mathrm{n}^{\mathrm{N}}$ 2:116-125.

SCHILLER, G. (1982) Significance of Bedrock as a site factor for aleppo pine. Forest Ecology and Management, 4:213-223.

SHEPHERD, K.R. (1986) Plantation silviculture Forestry Sciences. Martinus Nijhoff Publishers, Dordrecht; pp. 322

SPECHT, R.L. (1988) Mediterranean-type ecosystems. A data source book. Kluwer Academic Publishers. Dordrecht. pp. 248

SPURR, S.H. BARNES, B.V. (1980) Forest ecology. John Wiley \& Sons; pp. 687. 